\title{
The Influence of the Relaxation Time on the Dynamic Hysteresis in Perovskite Solar Cells
}

\author{
Alexandra Palici ${ }^{1}$, George Alexandru Nemnes ${ }^{2,3, \star}$, Cristina Besleaga ${ }^{1}$, Lucian Pintilie ${ }^{1}$, \\ Dragos-Victor Anghel ${ }^{3}$, loana Pintilie ${ }^{1}$, and Andrei Manolescu ${ }^{4}$ \\ ${ }^{1}$ National Institute of Materials Physics, Magurele 077125, Ilfov, Romania \\ ${ }^{2}$ University of Bucharest, Faculty of Physics, Materials and Devices for Electronics and Optoelectronics \\ Research Center, 077125 Magurele-Ilfov, Romania \\ ${ }^{3}$ Horia Hulubei National Institute for Physics and Nuclear Engineering, 077126 Magurele-llfov, Romania \\ ${ }^{4}$ School of Science and Engineering, Reykjavik University, Menntavegur 1, IS-101 Reykjavik, Iceland
}

\begin{abstract}
We investigate the dynamic behavior of perovskite solar cells by focusing on the relaxation time $\tau$, which corresponds to the slow de-polarization process from an initial bias pre-poled state. The dynamic electrical model (DEM) is employed for simulating the $\mathrm{J}-\mathrm{V}$ characteristics for different bias scan rates and pre-poling conditions. Depending on the sign of the initial polarization normal or inverted hysteresis may be induced. For fixed pre-poling conditions, the relaxation time, in relation to the bias scan rate, governs the magnitude of the dynamic hysteresis. In the limit of large $\tau$ the hysteretic effects are vanishing for the typical range of bias scan rates considered, while for very small $\tau$ the hysteresis is significant only when it is comparable with the measurement time interval.
\end{abstract}

\section{Introduction}

The dynamic hysteresis [1] observed in perovskite solar cells (PSCs) has become a notorious hallmark. Yet recently an increasing number of studies reported PSCs with diminished hysteresis and relatively large power conversion efficiencies (PCEs). However, knowing that the dynamic effects strongly depend on solar cell pre-conditioning and measurement history, unambigous comparisons regarding the magnitude of the observed hysteresis are rather difficult. Presently, a rather large consensus [2] has been reached concerning the origin of the dynamic hysteresis, which points to the ion migration within the perovskite layer as the main reason for the slow process, characterized by a relaxation time $\tau$ which is typically in the order of seconds. In particular, iodine migration was already evidenced in several reports, as well as in our own samples [3].

In this context, we investigate the hysteretic behavior dependence on the relaxation time $\tau$ at different bias pre-poling conditions. We base our analysis on the dynamic electrical model (DEM) [4], which is able to accurately reproduce the experimental J-V characteristics. In particular, a unified picture comprising normal (NH) and inverted (IH) hysteresis in the same device structure subject to different bias pre-conditioning was recently given by DEM, validated by J-V measurements [5].

^e-mail: nemnes@solid.fizica.unibuc.ro 
DEM provides a time dependent description of the J-V characteristics, by solving the coupled system of differential equations with given initial conditions as described in detail in Refs. $[4,5]$ :

$$
\begin{aligned}
-R_{s} C_{0} \frac{\partial I}{\partial t} & =I_{s}\left(e^{\frac{q\left(V+I R_{s}\right)}{n k_{B} T}}-1\right)+\left(\frac{R_{s}}{R_{s h}}+1\right) I+\frac{V}{R_{s h}}+C_{0} \frac{\partial V}{\partial t}+\mathcal{A} \frac{\partial P_{n l}}{\partial t}-I_{p h}, \\
\frac{\partial P_{n l}}{\partial t} & =-\frac{P_{n l}(t)-P_{n l, \infty}\left(U_{c}(t)\right)}{\tau} .
\end{aligned}
$$

Thus, the time dependence of the current and non-linear polarization, $I(t)$ and $P_{n l}(t)$, are obtained for an input voltage scan $V(t)$.

The core assumption of DEM is enclosed in Eq. (2), which describes the time evolution of $P_{n l}$ using a single relaxation time $\tau$. A condition of the same type was used for the surface polarization voltage in the model proposed by Ravishankar et al. [6], where it was conjectured that the cation migration together with an accumulation of holes is responsible for the hysteretic effects. According to Eq. (2) the non-linear polarization $P_{n l}$ follows, within the relaxation time $\tau$, the equilibrium value $P_{n l, \infty}=\left(U_{c} / V_{o c}\right) P_{\infty}$, which is a bias dependent quantity, where $U_{c}=V+I R_{s}$. The notations are the same as in Refs. [4, 5].

We assume the relaxation time is set by the morphology of the interfaces and layers of the PSC, i.e. it is a structure dependent quantity, although it may be further influenced by other factors like prepoling conditions, temperature etc. DEM suitably describes the experimental J-V characteristics using a single relaxation time, although multiple polarization mechanisms with different relaxation times may be present, e.g. induced by the drifting of several ionic species. If these individual contributions can be differentiated, then Eq. (2) may be extended to accomodate a more detailed description. In the next section we discuss the dynamic hysteretic effects with the focus on changing $\tau$, for two different initial polarizations, which correspond to normal and inverted hysteresis.

\section{Dynamic hysteretic effects - the $\tau$ dependence}

We simulate the dynamic J-V characteristics following the measurement protocol introduced in Ref. [5], i.e. the samples are pre-poled with an initial polarization $P_{0}$ and subsequently a reverse-forward (R-F) bias scan with constant rate $\alpha=\partial V / \partial t$ is performed. Figure 1 shows R-F scans corresponding to four different relaxation times, $\tau=5,10,20,40 \mathrm{~s}$, for an initial polarization $P_{0}=5 P_{\infty}$ and the same set of bias scan rates, $\alpha=5,20,100,1000 \mathrm{mV} / \mathrm{s}$. Since $P_{0}>0$ for each relaxation time, normal hysteresis behavior is obtained for any $\tau$ as explained in Ref. [4]: the hysteresis is diminished for very small and very large scan rates, while the short-circuit current $I_{s c}$ increases with $\alpha$. However, modifying $\tau$ the overall picture regarding the dynamic hysteretic effects is changing.

In the limit of large relaxation times $(\tau=40 \mathrm{~s})$ the hysteresis is diminished for all bias scan rates, while the increase of $I_{s c}$ is minimal. Setting $\tau \rightarrow \infty$ in Eq. (2), the current corresponding to the variation of the non-linear polarization vanishes, i.e. $\partial P_{n l} / \partial t \rightarrow 0$, and, in the limit of small geometrical capacitance $C_{0} \rightarrow 0$, the stationary case is recovered. Therefore, for a typical range of bias scan rates considered here and relatively large $\tau$, the $\mathrm{J}-\mathrm{V}$ characteristics are closing in to the stationary case, roughly corresponding to the $5 \mathrm{mV} / \mathrm{s} \mathrm{J}-\mathrm{V}$ characteristics.

Decreasing the relaxation time and keeping $\alpha$ fixed, $I_{s c}$ increases. Furthermore, for fixed, finite value of $\tau$, the hysteresis has a nonmonotonic dependence on $\alpha$, reaching a maximum when the reverse/forward scan time $V_{o c} / \alpha$ becomes comparable with $\tau$. This behavior can be explained as follows. For smaller $\tau$ values, $\partial P_{n l} / \partial t$ is getting larger at given $\alpha$, enhancing the output current. Increasing $\alpha$, the hysteresis reaches a maximum, after which is decreasing. For very fast bias scans, the non-linear polarization remains practically unchanged, $P_{n l} \approx P_{0}$. 


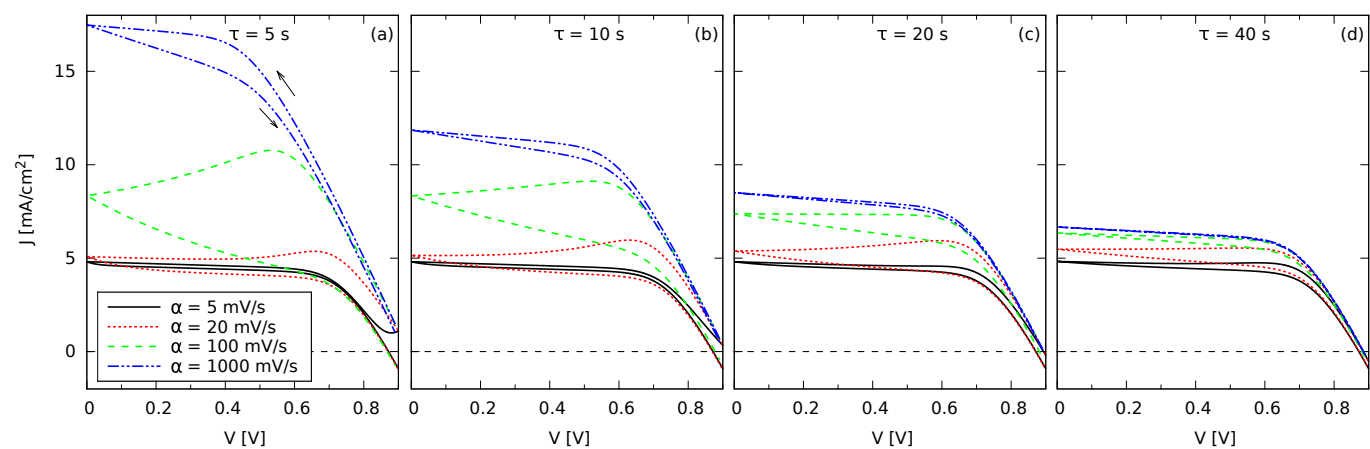

Figure 1. Normal hysteresis effects under changing the relaxation time $\tau: 5 \mathrm{~s}(\mathrm{a}), 10 \mathrm{~s}(\mathrm{~b}), 20 \mathrm{~s}(\mathrm{c}), 40 \mathrm{~s}$ (d). The bias scan rates are $\alpha=5,20,100,1000 \mathrm{mV} / \mathrm{s}$. The initial polarization $P_{0}=5 P_{\infty}$. The DEM parameters are: the series resistance $R_{s}=250 \Omega$, the shunt resistance $R_{s h}=20 \mathrm{k} \Omega$, the diode saturation current $I_{s}=0.1 \mathrm{pA}$, the diode ideality factor $n=1.53$, the photogenerated current $I_{p h}=0.43 \mathrm{~mA}$, the device area $\mathcal{A}=0.125 \mathrm{~cm}^{2}$, the open-circuit voltage $V_{o c}=0.9 \mathrm{~V}$ (stationary case) and the open-circuit polarization $P_{\infty}=12 \mathrm{mC} / \mathrm{cm}^{2}$. The arrows indicate the $\mathrm{NH}$ behavior.

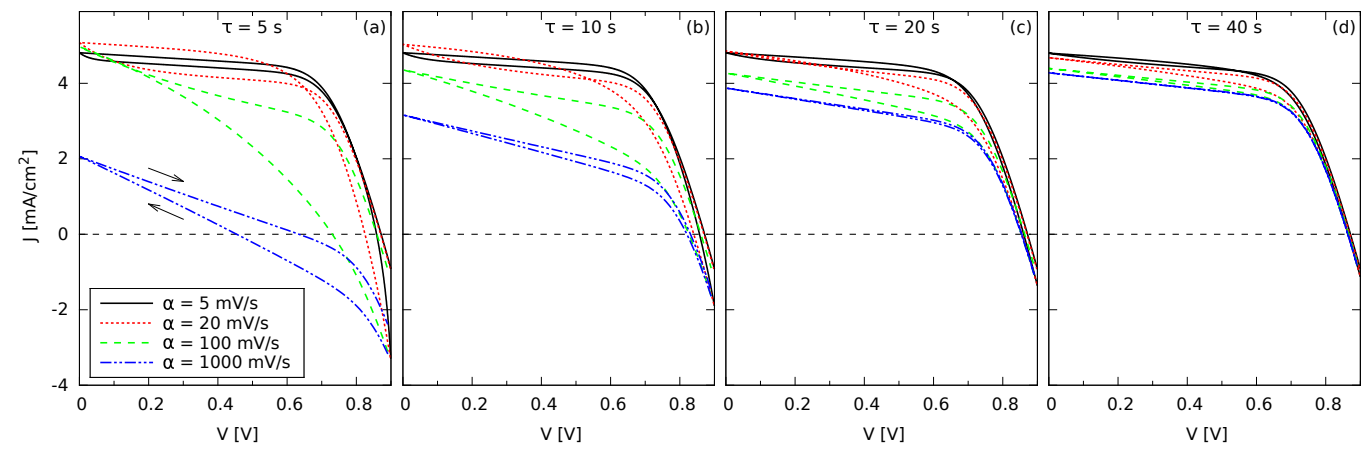

Figure 2. Inverted hysteresis effects obtained for the same set of parameters as in Fig. 1, apart from the initial polarization $P_{0}=-P_{\infty}$. The IH is present for $V>V_{c}$, i.e. to the right of the crossing point, while NH is visible for $V<V_{c}$ in some instances.

A different situation occurs when the initial polarization is negative, $P_{0}<0$, and an inverted hysteresis is evidenced, i.e. the current in forward scan is larger than in reverse [5]. Assuming the same parameters of the DEM and changing only $P_{0}=-P_{\infty}$, we obtain the $\mathrm{J}-\mathrm{V}$ characteristics depicted in Fig. 2. With respect to $\tau$ variations, there is an overall similar behavior as in the $\mathrm{NH}$ case. However, under IH pre-poling conditions, the resulting $I_{s c}$ decreases for higher bias scan rates, except for small $\tau$ values and slowest scan rates of 5 and $20 \mathrm{mV} / \mathrm{s}$. To explain this, one should note that for these two scan rates, a relatively small $\left|P_{0}\right|$ induces a mixed hyteresis, characterized by a crossing point at bias $V_{c}$, which separates the $\mathrm{IH}$ behavior at large biases from the $\mathrm{NH}$ occuring at smaller ones, e.g. near short-circuit. For $\alpha=5$ or $20 \mathrm{mV} / \mathrm{s}, P_{0}$ decays significantly during the reverse scan particularly for small $\tau$ and subsequently $P_{n l}$ follows $P_{n l, \infty}\left(U_{c}\right)$ as the bias is further decreased, resulting in $\mathrm{NH}$ behavior at small bias. By contrast, for $\alpha=100$ or $1000 \mathrm{mV} / \mathrm{s}, P_{n l}$ changes are similar on both reverse and forward scans, while $\partial P_{n l} / \partial t$ becomes larger, so that eventually the hysteresis becomes 
completely inverted and $I_{s c}$ decreases. Similarly, increasing $\tau$ we have $P_{n l} \approx P_{0}$ and the completely IH behavior is obtained at even lower scan rates, while the hysteresis is diminished.

From experimental point of view, achieving a certain initial polarization depends on the structure of the PCS, as well as on the pre-poling conditions, like poling bias $V_{p o l}$ and poling time $t_{p o l}$ [5]. In terms of relaxation times, it is more dificult to obtain the same $P_{0}$ as $\tau$ increases. For large $\tau$ values, the PSC is hysteresis-less, while for very short ones a fast enough scan rate can produce an apparently hysteresis-less behavior, although far away from the stationary case. Probing the dynamic behavior of the PSCs by different bias scan rates and poling conditions is recommended for a correct evaluation of the stationary $\mathrm{J}-\mathrm{V}$ characteristics and can provide additional insight into the ion migration related degradation.

\section{Conclusions}

We investigated the hysteretic behavior of PSCs under the change of the relaxation time scale, which is a central quantity in the DEM. The two types of the hysteresis, normal or inverted, determined by the sign of the initial polarization are analyzed in this context, for a typical range of bias scan rates. In the case of large relaxation times the dynamic hysteresis is vanishing and the $\mathrm{J}-\mathrm{V}$ characteristics become close to the stationary case even for the very large scan rates considered. In contrast, for smaller relaxation time scales, the short-circuit current changes significantly, either increasing for $\mathrm{NH}$ case or being reduced for $\mathrm{IH}$ case. The hysteresis is enhanced at intermediate scan rates, while at very fast and very slow scan rates it is diminished. It is therefore worth noting that measuring the J-V characteristics at rather high bias scan rates could lead to an apparent hysteresis-less behavior, with relatively large PCE, although far away from the stationary characteristics. In addition, the hysteretic behavior is consistently evaluated if the reverse and forward scans are correlated. Otherwise, independent scans having presumably different initial polarizations could yield a rather unpredictable and irreproducible results. The experimental determination of the specific relaxation times and the investigation of the dynamic effects, monitored over time, may also provide further insight into ion migration induced degradation of the PSCs.

\section{Acknowledgement}

This work was supported by Romania-JINR cooperation project JINR Order 220/10.04.2017, pp. 24 and pp. 94 and EEA Financial Mechanism 2009-2014 under project contract no. 8SEE/30.06.2014.

\section{References}

[1] H. J. Snaith, A. Abate, J. M. Ball et al., J. Phys. Chem. Lett. 5, 1511 (2014)

[2] W. Tress, J. Phys. Chem. Lett. 8, 3106 (2017)

[3] Cristina Besleaga, Laura Elena Abramiuc, Viorica Stancu, A. G. Tomulescu, M. Sima, Liliana Trinca, N. Plugaru, L. Pintilie, G. A. Nemnes, Mihaiela Iliescu, H. G. Svavarsson, A. Manolescu and Ioana Pintilie, J. Phys. Chem. Lett. 7, 5168 (2016)

[4] G. A. Nemnes, Cristina Besleaga, A. G. Tomulescu, Ioana Pintilie, L. Pintilie, K. Torfason, A. Manolescu, Sol. Energy Mater. Sol. Cells 159, 197 (2017)

[5] G. A. Nemnes, Cristina Besleaga, Viorica Stancu, Daniela Emilia Dogaru, Lucia Nicoleta Leonat, L. Pintilie, K. Torfason, M. Ilkov, A. Manolescu, Ioana Pintilie, J. Phys. Chem. C 121, 11207 (2017)

[6] S. Ravishankar, O. Almora, C. Echeverria-Arrondo, E. Ghahremanirad, C. Aranda, A. Guerrero, F. Fabregat-Santiago, A. Zaban, G. Garcia-Belmonte, and J. Bisquert, J. Phys. Chem. Lett. 8, 915 (2017) 